\title{
Omega ( $\omega)$-3 Polyunsaturated Fatty Acids (PUFAs) Derived from Microorganisms and Their Role in Human Health
}

\author{
Naveena K C, Ramalingappa B* \\ Department of Microbiology, Davangere University, Davangere-577007, Karnataka, India.
}

*Corresponding Author: Ramalingappa B, Department of Microbiology, Davangere University, Davangere-577007, Karnataka, India.

\begin{abstract}
Oleaginous microorganisms (Thraustochytrids) are typical marine fungi that produce docosahexaenoic acid (DHA) and eicosapentaenoic acid (EPA), which are $\omega-3$ polyunsaturated fatty acids (PUFAs). The use of microbial DHA and EPAs is rapidly improving human health and therefore arousing several clinical trials. To cultivate Thraustochytrids, the technique used is very important. A number of researches suggested that DHA and EPA ameliorate many health problems including cardiovascular disorders, rheumatoid arthritis, different types of cancers and alzheimer's disease and so many, which are thus used for pharmacological and as well as nutraceutical purpose.
\end{abstract}

Keywords: Thraustochytrids, docosahexaenoic acid (DHA), eicosapentaenoic acid (EPA), oleaginous microorganisms, omega-3 fatty acids.

\section{INTRODUCTION}

Lipids are naturally occurring compounds obtained from microorganisms and plants, which are insoluble in water and organic compounds but soluble in organic solvents such as alcohol and ether [1], they pocesses some naturally occurring fatty acids (Table 1). Lipids include oils, fats, wax, sterols, glycolipids, monoglycerides, diglycerides, triglycerides, phospholipids and some other related compounds, which are utilized by living cells [2]. Microbial lipids containing high proportion of polyunsaturated fatty acids (PUFAs) such as omega-3, omega 6 and omega-9 polyunsaturated fatty acids of nutritional and pharmaceutical importance and microbial lipids have similar composition with edible and non-edible oils obtained from plants and animals known as single cell oil (SCOs). PUFAs have diverse functions in living cells and influence membrane composition and function, eicosanoid synthesis, cellular signaling and regulation of gene expression [3, 4].

Lipids are rich in PUFAs, which are present in plants, animals and microorganisms in sufficient quantities, they are known as specialty lipids. Their production has been known for so many years. For the last two decades, many attempts have been made to understand the process of liquid accumulation in oleaginous species. All microorganisms do not have the capacity to accumulate lipid in the form of triacylglycerol but some of prokaryotic and eukaryotic microorganisms can accumulate triacylglycerol as cellular storage from 20 to $70 \%$ of the dry biomass and are known as oleaginous species [5, 6]. Demands for PUFAs are constantly increasing while the sources producing them are not increasing at the same rate [4]. Various alternative sources are being explored which yield these essential demands for health, leading to the screening of new strains and better understanding of lipid production in microorganisms. It is found that yeast, fungi, and a few bacteria are the major oil accumulating microorganisms and produce extractable oil. Omega-3 fatty acids are also known as $\dot{\omega}-3$ fatty acids or n-3 fatty acids, these are polyunsaturated fatty acids obtained by microbes that they have several double bonds in the chemical structure. The major nutraceuticals of these lipid classes are arachidonic acid (ARA), docosahexaenoic acid (DHA) and eicosapetaenoic acid (EPA). Plants are not able to synthesis these long chain unsaturated fats with the exception of gamma-linolenic acid (GLA) which is traditionally created from the seeds of night primrose, borage and blackcurrant. As a choice to plant and animal based oils, investigate is being focusssed on screening and isolation of new oleaginous microorganisms, which has investigated certain parasites, marine microscopic organisms, heterotrophic and phototropic small scale green growth and greeneries incorporating microbial oils having long chain unsaturated fats [7]. 
Omega-3 polyunsaturated fats (long chain) are eicosapentaenoic acid (EPA) and docosahexaenoic acid (DHA) are recommended as advantageous components pharmaceutically and nutraceutically [8] and are also prescribed as food added substance. Docosahexaenoic acid is useful as it prevents sicknesses of cardiovascular, growths, Alzheimer's disease, and joint pain [9]. Omega-3 unsaturated fats and specifically DHA are important in a child's brain development and retinal improvement. In this way, they need to be added to the eating regular with a specific goal to keep up one's physical and mental capacities. Really, the Dietetic Association of America and Canada Dieticians formally approve that 20 to $35 \%$ of our eating routine ought to originate from dietary fat, with an accentuation on using omega-3 unsaturated fats [10]. The unicellular eukaryotic marine protists group of Thraustochytrids incorporates the genera of Aurantiochytrium, Parietichytrium, Schizochytrium and Thraustochytrium. Thraustochytrids are thought to be far superior in the production and accumulation of PUFAs, as they have a lot of DHA and n-6 docosapentaenoic acid (C22:5n-6) with minimal cross contamination with EPA or arachidonic acid $(\mathrm{C} 20: 4 n-6)[11,12]$. This is due to the presence of PUFA synthase in them that supplements the standard pathway for unsaturated fat/squalene creation.

It is discovered that grown-ups in the most note worthy quarter have around $4 \mathrm{~mm} \mathrm{Hg}$ bring down systolic and $2 \mathrm{~mm} \mathrm{Hg}$ bring down diastolic circulatory strain in contrast with those with minimum omega-3 unsaturated fats in their blood [13]. By and large, higher omega-3 unsaturated fats in the blood simply bring down both systolic and diastolic circulatory strain. This suggests that diets rich in omega-3 sustenance could prevent hypertension [13]. The researchers measured circulating PUFAs in the blood samples of individuals from eight countries in Europe, who were part of EPIC-Interact, the world's largest study of new-onset type 2 diabetes. They compared baseline levels of 11 different PUFAs (4 long-chain omega-3 PUFAs and 7 omega-6 PUFAs) between 12,132 individuals who subsequently developed type 2 diabetes over a follow-up period of approximately 10 years $(n=12,132)$ and 15,919 individuals in a sub-cohort representative of the whole EPIC study population [14], n-3 polyunsaturated fatty acids of EPA and DHA have anti-inflammatory, anti atherosclerotic and neuronal protective functions and may prevent dementia [15]. They have many health benefits including the prevention of cardio metabolic and some disease and as well as reduce inflammation, and also play an important role in metabolism of bone to prevent bone loss and reduce osteoporosis risk [16]. EPA and DHA are shown to reduce plasma triglyceride levels as well as have calming impacts and enhance endothelial capacity, all of which mediate against atherogenic impacts [17]. Some valuable impacts of EPA and DHA are lowering blood lipids, reducing invulnerable wounds, repressing thrombogenesis, enhancing intellectual capacity, mitigating dejection and controlling tumor growth [18]. Long-chain omega-3 PUFAs can diminish platelet total and accordingly thrombus development. Besides, omega-3 PUFAs have a calming activity, which may help to balance out atherosclerotic plaques, in this manner keeping their break [19].

\section{Classification}

Lipids are broadly classified in to simple, complex and derived lipids, which are further subdivided into different groups.

\subsection{Simple Lipid}

These are esters of fatty acids with various alcohols including:

a). Fats and oils: These are esters of fatty acids with glycerol, the only physical difference between fats and oils is that fats are in the form of solid state, while oils are in the form of liquid state at room temperature [2].

b). Waxes: These are long chain esters of fatty acids along with alcohols other than glycerol. These alcohols may be alicyclic or aliphatic. In waxes acetyl alcohol is mostly determined [2].

\subsection{Compound/Complex Lipid}

These are esters of fatty acids containing groups in addition to an alcohol and fatty acid.

a. Phospholipids: These are lipids containing unsaturated fats and alcohol, a phosphoric acid deposit. They have nitrogen containing bases and different substituents, e.g. in "glycerophopholipids" the alcohol is glycerol and in 'sphingo-phospholipids' the alcohol is sphingosine [20].

b. Glycolipids: These are lipids containing unsaturated fat, sphingosine, sugar and nitrogenous base. Glycerol and phosphate are absent (e.g. cerebrosides and gangliosides). 
c. Lipoproteins: These include macromolecular complexes of lipids with protein.

d. Other complex lipids: These include lipids such as sulfolipids, and amino lipids [20].

\subsection{Precursor and Derived Lipids}

These include fatty acids, glycerol, steroids, different alcohols, fatty aldehydes, ketone bodies, hydrocarbons, lipid-dissolvable vitamins and hormones [20].

\section{Polyunsaturated (OMega-3) FatTy Acids}

Omega-3 polyunsaturated fatty acids are type of fatty acids having different double bonds three atoms away from the terminal methyl group, such as EPA and DHA (eicosapentaenoic acid 20:5 and docosahexaenoic acid 22:6) and $\alpha$ - linolenic acid [21] (Table 2). Generally omega-3 polyunsaturated fat plays important roles in the human body including control of some specific genes [22]. Omega-3 fatty acids change the processes in the body, for example biosynthesis of fatty acids and transport of cholesterol; also they have the capacity to regulate the structure, permeability and various aspects of cell membranes. They are needed by each organ of the body for normal functioning; $\omega-3$ fats cannot be synthesized in most animals and humans, so they must be obtained from diet [23].

Table1. Some naturally occurring fatty acids.

\begin{tabular}{|c|c|c|}
\hline Common name & Short name & Systematic name \\
\hline \multicolumn{3}{|l|}{ Saturated fatty acid } \\
\hline Palmitic acid & 12:0 & Dodecanoic acid \\
\hline Lauric acid & $16: 0$ & Hexadecanoic acid \\
\hline Myristic acid & 14:0 & Tetradecanoic acid \\
\hline Stearic acid & 18:0 & Octadecanoic acid \\
\hline \multicolumn{3}{|c|}{ Monounsaturated fatty acid } \\
\hline Oleic acid & $\Delta 918: 1$ & $\Delta$ 9- Octadecenoic acid \\
\hline Palmitoleic acid & $\Delta 916: 1$ & $\Delta$ 9-Hexadecenoic acid \\
\hline \multicolumn{3}{|c|}{$\omega-6$ Polyunsaturated fatty acid } \\
\hline Arachidonic acid & $\omega-620: 4$ & $\Delta 5, \Delta 8, \Delta 11, \Delta 14$ Eicosatetraenoic acid \\
\hline Linoleic acid & $\omega-618: 2$ & $\Delta 9, \Delta 12$ Octadecadienoic acid \\
\hline \multicolumn{3}{|c|}{$\omega-3$ Polyunsaturated fatty acid } \\
\hline$\alpha$ Linolenic acid & $\omega-318: 3$ & $\Delta 9, \Delta 12, \Delta 15$ Octadecatrienoic acid \\
\hline Docosahexaenoic acid & $\omega-322: 6$ & $\Delta 4, \Delta 7, \Delta 10, \Delta 13, \Delta 16, \Delta 19$ Docosahexaenoic acid \\
\hline Eicosapentaenoic acid & $\omega-320: 5$ & $\Delta 5, \Delta 8, \Delta 11, \Delta 14, \Delta 17$ Eicosapentaenoicacid \\
\hline
\end{tabular}

Source: Adopted from [1]

Table2. Omega-3 polyunsaturated fatty acids.

\begin{tabular}{|l|c|c|}
\hline Common name & Systematic name & Short name \\
\hline \multirow{2}{*}{ Docosahexaenoic acid } & $\Delta 4, \Delta 7, \Delta 10, \Delta 13, \Delta 16, \Delta 19$-Docosahexaenoic acid & $\omega-322: 6$ \\
\cline { 2 - 3 } & $\Delta 5, \Delta 8, \Delta 11, \Delta 14, \Delta 17, \Delta 20$-Tetracosahexaenoic acid & $\omega-324: 6$ \\
\hline & & \\
\hline \multirow{2}{*}{ Eicosapentaenoic acid } & $\Delta 5, \Delta 8, \Delta 11, \Delta 14, \Delta 17$-Eicosapentaenoic acid & $\omega-320: 5$ \\
\cline { 2 - 3 } & $\Delta-7, \Delta 10, \Delta 13, \Delta 16, \Delta 19$-Docosapentaenoic acid & \\
\hline \multirow{3}{*}{$\alpha$ - Linolenic acid } & $\Delta 9, \Delta 12, \Delta 15$-Octadecatrienoic acid & $\omega-318: 3$ \\
\cline { 2 - 3 } & $\Delta 6, \Delta 9, \Delta 12, \Delta 15$-Octadecatetraenoic acid & $\omega-318: 4$ \\
\cline { 2 - 3 } & $\Delta 8, \Delta 11, \Delta 14, \Delta 17, \Delta 20$-Tetracosahexaenoic acid & $\omega-320: 4$ \\
\hline
\end{tabular}

Source: Adopted from [21].

\section{OMega-3 Polyunsaturated Fatty Acids (Pufas) Health Benefits}

The human body is designed to live on an eating routine with a balance between omega- 3 and omega6 unsaturated fats, approximately close to a proportion of 1:1[24]. The typical American diet, however, has a proportion of omega- 6 to omega-3 unsaturated fats of 14:1 [25]. Omega-3 unsaturated fats are vital for humans. 


\subsection{Cardiovascular System}

The coronary heart disease is most commonly cause of cardiac arrest and huge blood loss, lack of oxygen, very low level of potassium, heart failure and exercise of intense physical are the less commonly causes. A long QT syndrome increased by number of inherited disorders. The underlying heart beat is frequently ventricular fibrillation. While heart failure might be brought by heart attack or heart failure, these are not the same [26].

Three epidemiologic reviews directed in the United States are additionally predictable with this outcome. In the examination of 80 grown-ups who experienced essential heart failure and 108 strong grown-ups, some researchers found that the EPA and DHA substances of erythrocyte layer are connected to essential heart failure [27]. Scientists inspected the hazard factors by taking the blood tests of 14,916 sound guys, and after 17 years, examined the information of 94 guys who passed on as a result of coronary illness. The study showed that their omega- 3 unsaturated fat substance was low [28]. Also some researchers distinguished that EPA blood and DHA substances have a connection with lethal ischemic coronary illness [29].

Some investigation revealed that ingesting 1 to $1.5 \mathrm{~g}$ of DHA and EPA lessens non-cholesterol blood non partisan fats by 25 to $30 \%$ [30]. Moreover, some examination result demonstrated that fish oil, which has a high quantity of DHA and EPA, stifles the action of endothelial lipase that expands the grouping of plasma high-thickness lipoprotein cholesterol. A current xmeta-investigation contemplate revealed that the adequate admission of $\alpha$-linolenic acid (ALA), which is an omega-3 arrangement unsaturated fat, additionally decreases the beginning recurrence of cardiovascular ailments [31].

\subsection{Cancer}

A term for disease in which abnormal cells divide without control and can invade nearby tissues. Cancer cells can also spread to other parts of the body through the blood and lymph systems. There are several main types of cancers.

A few investigations have tended to the restorative impacts of omega-3 PUFAs in disease demonstrating that omega-3 PUFAs can adequacy and bearableness of chemotherapy [32]. There are clinical trials where DHA alone or blends of omega-3 PUFAs are being tried for growth anticipation, support, or treatment [33]. DHA as a treatment system is regularly joined with chemotherapeutic medications since DHA in all likelihood upgrades the cytotoxic impacts of these medications [34].

DHA may also have a positive effect on the prevention and treatment of cancer. DHA is in high levels in healthy cells protecting them from apoptosis, while DHA is lower in unhealthy cells inducing apoptosis [35]. Potential medication sharpening impacts of DHA and EPA have additionally been accounted for in various investigations to such an extent that low measures of these two FAs in blend with anticancer operators can bring about expanded affectability of tumor cells to hostile and neoplastic operators even in some medications for safe cell [36].

\subsubsection{Breast Cancer}

In human, breast cancer is the most common cancer. It recorded in worldwide more than one million of new cases per year [37]. High level administration of eicosapentaenoic acid and docosahexaenoic acid through diet was connected to a $25 \%$ decrease in bosom tumor repeat [38]. Most work assessing how EPA and DHA work to reduce breast cancer risk has been performed in in vitro or in transgenic mouse models and is far from conclusion. Nonetheless, the transcendent components are believed to be: A lessening in proinflammatory eicosanoids and an expansion in aggravation settling subordinates as point by point before hand, a decrease in oncogenic protein motioning through disturbance of plasma film lipid pontoons; a decrease in cytokine generation; and an expansion in apoptosis following enactment of plasma layer GRP120 protein receptor, alongside actuation of peroxisome proliferators-initiated receptor gamma pieces atomic factor- $\mathrm{\kappa B}$ translocation to the core [39, 40]. A solid relationship exists between eating routine, over night and danger of essential breast cancer what's more, its repeat [41].

\subsubsection{Skin Cancer}

One investigation assessed the impact of omega-3 unsaturated fat on the frequency of skin growth among male human services experts. This investigation surveyed the frequency of basal cell 
carcinoma in respect to the utilization of omega-3 unsaturated fat. In respect to members in the least quartile of omega-3 fat utilization, those in the most elevated quartile of utilization had a little yet factually huge increment in the danger of basal cell carcinoma (RR, 1.13; 95\% CI, 1.01-1.27) [42].

\subsubsection{Stomach Cancer}

Researchers have recognized and assessed the impact of omega- 3 unsaturated fat on the rate of stomach malignancy. This study evaluated its occurrence with respect to fish utilization and discovered there was no relationship with the frequency of stomach malignancy [43].

\subsection{Rheumatoid Arthritis}

This is an autoimmune disease, which causes joint inflammation. Some studies and several researchers have found that DHA+EPA can reduce rheumatoid symptoms, morning stiffness and joint pain. Other rheumatoid trials of omega-3 supplementation in rheumatoid arthritis have revealed decrease in terms of morning firmness, time to weakness and utilization of non-steroidal calming drugs [44]. There were outcomes from a one year randomized controlled trial of high and low dosage angle oil supplement in patients who had rheumatoid arthritis, with high amounts of plasma phospholipids, EPA and DHA and high probability of accomplishing abatement based on American College of Rheumatology criteria [45].

Now a day's literature indicates supplementation of omega-3 PUFA reduces inflammatory, cytokines and eicosanoids in patients with rheumatoid arthritis. Subsequently, these impacts ought to lessen torment and ligament obliteration which, in turn, may lead patients to control their utilization of pain controlling drugs. Randomized controlled trials of $\omega 3$ fatty acids (at measurements in the vicinity of 1 and $7 \mathrm{~g}$ every day) in RA have announced enhancements in a few clinical results including reduced length of morning solidness, decreased number of delicate or swollen joints, diminished joint agony, decreased time to weariness, expanded hold quality and diminished utilization of agony controlling medications [46].

\subsection{Alzheimer's Disease}

Loss of memory is an indication of Alzheimer's disease, which is dynamic and makes the patient unable to fend for his or herself and inevitably die [47]. Neuron membrane plasma lipid has large amounts of DHA, it plays a role in nervous system function.

A case-control comprising 148 patients with subjective weakness [Mini-Mental State Examination (MMSE) score $<24$ ] and 45 control patients (MMSE score $\geq 24$ ) demonstrated that serum cholesterol ester-EPA and - DHA levels were altogether lower ( $\mathrm{P}<0.05$ and $\mathrm{P}<0.001$, separately) in all MMSE score quartiles of patients with $\mathrm{AD}$ contrasted and control esteems [48]. Another investigation found that an eating routine containing high omega- 3 unsaturated fats (plate of mixed green dressing, nuts, angle, tomatoes, poultry, cruciferous vegetables, organic products, dull and green verdant vegetables), and a bring down admission of sustenance low in omega-3 unsaturated fats (high-fat dairy items, red meat, organ meat, margarine) was firmly linked to a lower $\mathrm{AD}$ chance [49]. Patients' weight essentially expanded by $0.7 \mathrm{~kg}$ in the EPA+DHA treatment at $6 \mathrm{mo}(\mathrm{P}=0.02)$ and by $1.4 \mathrm{~kg}$ at $12 \mathrm{mo}$ $(\mathrm{P}<0.001)$. It was watched primarily in patients with a BMI $<23$ at the beginning of the investigation [50]. This implies those patients with a lower BMI specially put on weight in contrast with those patients with a higher BMI.

\section{Pharmaceutical And Nutraceutical Applications}

Confirmation of the conceivable medicinal impacts of PUFA insufficiency has, combined with the developing acknowledgment of pharma foods (nutraceuticals) by customers conveyed these mixes considering the sustenance and pharmaceutical organizations. This has been snappy to endeavor showcases in biomedical and pharma food enclosures [51]. A mixed bag of strong PUFA lipids are accessible for restorative uses, running from anti- aging, calming, anti cholesterolaemic and anticancer medications to immune stimulant and immune suppressant therapeutics (Table 3), despite the fact that their efficacies are yet to be demonstrated. Unregulated applications of esters, glycerides and phospholipids, for example, nutraceutical added substances for sustenance, wholesome formulae and beautifying agents' fixings have likewise expanded. However, the most clear business effect of PUFAs has been in wellbeing supplements, with a large group of plant and fish determined GLA, EPA and DHA items now being accessible in the commercial center for uncontrolled dietary 
utilization [51, 52]. According to cohort and organization, intakes of docosahexaenoic acid and eicosapentaenoic acid (EPA+DHA) (Table. 4).

Table3. Nutraceutical and biomedical applications of commercial polyunsaturated fatty acids.

\begin{tabular}{|c|c|c|}
\hline Application & PUFA product & Reference \\
\hline Mitigating and immunosuppressant helpful & EPA and DHA oils & {$[53]$} \\
\hline Thrombolytic and anti-atherosclerotic chocolates & EPA/DHA fats/oils & {$[54]$} \\
\hline Therapeutics for diarrhea & ALA/EPA/DHA oils & {$[55]$} \\
\hline Presurgery immunostimulants & EPA/DHA oils & {$[56]$} \\
\hline Nutraceutical added substances for prepared food. & EPA, DHA and AA oils & {$[57]$} \\
\hline
\end{tabular}

ALA: $\alpha$-Linolenic acid. AA: arachidonic acid.

Table4. Intake recomennndation of EPA and DHA by cohort and organization.

\begin{tabular}{|c|c|c|}
\hline Cohort & Source & EPA+DHA (g) \\
\hline \multicolumn{3}{|l|}{ General health } \\
\hline Nutrition and Diet Academy & Adults & $\geq 0.5$ \\
\hline Heart Association of America & Adults without CHD & 0.5 \\
\hline Food Safety Agency of Europe & Adults & $\geq 0.25$ \\
\hline \multicolumn{3}{|c|}{ Heart disease and inflammatory disorder } \\
\hline Heart Association of America & CHD & 1 \\
\hline Heart Association of America & Patients having high TG & $1.8-4$ \\
\hline \multicolumn{3}{|l|}{ Pregnancy } \\
\hline Food Safety Agency of Europe & Lactating/pregnant & $\geq 0.25$ \\
\hline The Study of fatty acids and lipids of internati & Lactating/pregnant & $\geq 0.5$ \\
\hline
\end{tabular}

CHD: Coronary heart disease. TG: triacylglycerol.

Source: Adapted from [43].

\section{Conclusion and Future Prospective}

The use of $\omega$-3 PUFAs in our eating regimen, application of PUFAs in different fields in addition their importance in wellbeing and dietary pre requisites supports the chase for more suitable wellsprings of these mixes. It has impact on human's wellbeing as microbial oils (EPA, DHA and ARA); consequently these supplements are broadly acknowledged by people generally. Their administration has led to the introduction of PUFA enhanced items. The expanded utilization of PUFAs in human eating routine has without a doubt significant future potential business for PUFAs. Omega-3 PUFAs EPA and DHA are important to humans' wellbeing and are a dietary need discovered predominantly in fish and microorganisms like Thraustochytrids sp. EPA and DHA are fundamental for fitting fetal advancement and supplementation during pregnancy; also they enhance cardiovascular capacity. Furthermore, they are identified with heftiness, malignancy and joint inflammation.

Specialists have an extraordinary chance to find numerous and unidentified marine microorganisms fit for creating more elevated amounts of $\omega-3$ unsaturated fats and other profitable items. The creators proposed to give extra- data that reinforce the biosynthetic pathway of PUFA followed in marine microorganisms. One of the fundamental difficulties in creating ideal society conditions for developing marine organisms that produce elevated amounts of DHA and EPA contrasts with the set number of industrially helpful species accessible in the universal store.

At present, the cost of growing DHA and EPA is essentially higher than getting these unsaturated fats from fish oil. However, maturation empowers better control of EPA and DHA proportions and also diminishes contaminant levels. Thraustochytrids can create phospholipids and in this manner can possibly give a fermentable wellspring of oil like krill oil. Metabolic building could further enhance yields of DHA, EPA and different mixes from Thraustochytrids. The cost of fermentation can be minimized utilizing easy carbon sources, for example, glycerol, and the utilization of easy carbon hot spots for DHA generation from these living beings is needed. 


\section{REFERENCES}

[1] Arjuna A., Production of polyunsaturated fatty acids by fungi, International journal of Pharma and Biosciences. 5(3), 931-954 (2014).

[2] Sathyanarayana U, Harpers. Biochemistry, $26^{\text {th }}$ ed. 2003; PP. 129-131.

[3] Tapiero H., Ba GN., Couvreur P. and Tew KD., Polyunsaturated Fatty acids (PUFAs) and Eicosanoids in Human health and Pathologies, Alternative Medical Review. 56(5), 215-222 (2002).

[4] Wallis JG., Watts JL. and Browse J., Polyunsaturated Fatty acids Synthesis, Journal of Traditional Biochemical Science. 27(9), 467-470 (2002).

[5] Waltermann M., Luftmann H., Baumeister D., Kalscheuer R. and Steinbuchel A., Isolation and Characterization of triacylglycerol's and Other Storage Lipids, Journal of Microbiology. 146(5), 11431149 (2000).

[6] Ratledge C. and Wynn J., The Biochemistry and Molecular biology of lipid accumulation in oleaginous microorganisms, Journal of Advanced Applied Microbiology. 55(51), 1-51 (2002).

[7] Ahmed SU, Castro GR, Soccol CR, Soccol VT, Larroche C, Pandey A, Microbial lipids. Chapter 7, Comprehensive Food Fermentation Biotechnology- vol, 2. Asiatech Publishers Inc, New Delhi, India. 2010; Pp. 224-256.

[8] Dratz EA, Deese AJ, The role of docosahexaenoic acid in biological membranes examples from photoreceptors and model membrane bilayers. In: Simopoulos AP, Kifer RR, Martin RE, ed. Health effects of polyunsaturated fatty acids in seafood's. Academic Press; Orlando 1986; Pp. 319-351.

[9] Horrocks LA. and Yeo YK., Health benefits of docosahexaenoic acid (DHA), Journal of Pharmacological Research. 40(3), 211-225 (1999).

[10] Kris-Etherton PM. and Innis S., Position of the American Dietetic Association and Dietitians of Canada: dietary fatty acids, Journal of American Dietetic Association. 107(9), 1599-1611 (2007).

[11] Nakahara T., Yokochi T., Higashihara T., Tanaka S., Yaguchi T. and Honda D., Production of docosahexaenoic and docosapentaenoic acids by Schizochytrium sp. Isolated from Yap Islands, Journal of American Oil Chemist Society. 73(11), 1421-1426 (1996).

[12] Yaguchi T., Tanaka S., Yokochi T., Nakahara T. and Higashihara T., Production of high yields of docosahexaenoic acid by Schizochytrium sp. strain SR21. JAOCS, Journal of American Oil Chemist Society. 74, 1431-1434 (1997).

[13] American heart association. Diets rich in omega-3 fatty acids may help lower blood pressure in young, healthy adults (online) 2016. Available at www.sciencedaily.com.

[14] PLOS. Different types of PUFAs are associated with differential risks for type 2 diabetes (online) 2016. Available at www.sciencedaily.com.

[15] Yanai H., Effects of n-3 polyunsaturated fatty acids on dementia, Journal of clinical medicine research. 9(1), 1-9 (2017).

[16] Rajaram S., Yip EL., Reghunath R., Mohan S. and Sabate J., Suppliment on biomarkers of bone turnover in health adults, Journal of Nutrients. 9, 1162 (2017).

[17] Sakai C., Ishida M., Ohba H., Yamashita H., Uchida H., Yoshizumi M. and Ishida T., Fish oil omega-3 polyunsaturated fatty acids attenuate oxidative stress-induced DNA damage in vascular endothelial cells, Plos One. 12 (11), 1-12 (2017).

[18] Liu W., Xie X., Liu M., Zhang J., Liang W. and Chen X., Serum omega-3 polyunsaturated fatty acids and potential influence factors in elderly patients with multiple cardiovascular risk factors, Nature. 41598 (18), 19193-19195 (2018).

[19] Innes JK. and Philip C., The differential effects of eicosapentaenoic acid and docosahexaenoic acid on cardio metabolic risk factors, International Journal of Molecular Sciences. 19(2), 532 (2018).

[20] Botham KM, Mayers PA. Lipids of physiologic significance, $27^{\text {th }}$ ed. Harper's Illustrated Biochemistry 2006; Pp. 121-131.

[21] Swaff E. and Sijtsma I., Biotechnological production and applications of the omega-3 polyunsaturated fatty acid docosahexaenoic acid, Journal of Applied Microbial and Biotechnology. 64, 146-153 (2004).

[22] Sessler AM. and Ntambi JM., Polyunsaturated fatty acid regulation of gene expression, Journal of Nutrition. 128 (6), 923-926 (1998).

[23] Certik M. and Shimizu S., Biosynthesis and regulation of microbial polyunsaturated fatty acid production, Journal of Bioscience and Bioengineering. 87, 1-14 (1999).

[24] Simopoulos AP., The importance of the ratio of omega-6/omega-3 essential fatty acids, Biomedicine and Pharmacotherapy. 56(8), 365-379 (2002). 
[25] Marik PE. and Varon J., Omega-3 dietary supplements and the risk of cardiovascular events: A Systematic Review, Journal of Clinical Cardiology. 32(7), 365-372 (2009).

[26] Field. and John M., Cardiovascular Care and CPR. Lippincott Williams and Wilkins 2009. Pp. 11.

[27] Siscovick DS., Raghunathan TE., King I., Weinmann S., Wicklund KG. and Albright J., Dietary intake and cell membrane levels of long-chain n-3 polyunsaturated fatty acids and the risk of primary cardiac arrest, JAMA. 274(17), 1363-1367 (1995).

[28] Albert CM. Campos H., Stampfer MJ., Ridker PM., Manson JE. and Willett WC., Blood levels of longchain n-3 fatty acids and the risk of sudden death, New England Journal of Medicine.346(11),1113-1118 (2002).

[29] Lemaitre RN., King IB., Mozaffarian D., Kuller LH., Tracy RP. and Siscovick DS., N-3 polyunsaturated fatty acids, fatal ischemic heart disease and nonfatal myocardial infarction in older adults: the Cardiovascular Health Study, American Journal of Clinical Nutrition. 77 (2), 319-325 (2003).

[30] Harris WS., Rambjor GS., Windsor SL. and Diederich D., N-3 fatty acids and urinary excretion of nitric oxide metabolites in humans, American Journal of Clinical Nutrition. 65(2), 459-464 (1997).

[31] Pan A., Chen M., Chowdhury R., Wu JH., Sun Q. and Campos H., Recent process developments in solid state fermentations, Journal of Process Biochemistry. 27(2), 109-117 (2012).

[32] Bougnoux P., Hajjaji N., Ferrasson MN., Giraudeau B., Couet C. and Le Floch O., Improving outcome of chemotherapy of metastatic breast cancer by docosahexaenoic acid: a phase II trial, Brazilian Journal of Cancer. 101, 1978-1985 (2009).

[33] Berquin IM., Edwards IJ. And Chen YQ., Multi-targeted therapy of cancer by omega-3 fatty acids, Cancer Letter. 269,363-377 (2008).

[34] Nabavi SF., Bilotto S., Russo GL., Orhan IE., Habtemariam S. and Daglia M., Omega-3 polyunsaturated fatty acids and cancer: lessons learned from clinical trials, Cancer Metast. 34,359-380 (2015).

[35] Siddiqui RA., Harvey K. and Stillwell W., Anticancer properties of oxidation products of docosahexaenoic acid, Journal of Chemistry and Physics of Lipids. 153(1), 47-56 (2008).

[36] Moloudizargari M., Asghari MH. and Abdollahi M., Modifying exosome release in cancer therapy, Pharmacological Research. 134,246-256 (2018).

[37] Bougnoux P., Hajjaji Maheo K., Couet C. and Chevalier S., Fatty acids and breast cancer: sensitization to treatments and prevention of metastatic re-growth, Progress in Lipid Research. 49,76-86 (2010).

[38] Petterson R., Marine fatty acid intake is associated with breast cancer prognosis, Journal of Nutrition. 141(2), 201-206 (2011).

[39] Turk FH. and Chapkin RS., Membrane lipid raft organization is uniquely modified by $n-3$ polyunsaturated fatty acids, Pub med central. 88(1), 43-47 (2013).

[40] Carol J., Fabian Bruce F. and Kimler Stephen D., Omega-3 fatty acids for breast cancer prevention and survivorship, Journal of Biomed central. 17(1), 62 (2015).

[41] Molfino A., Amabile MI., Monti M., Arcieri S., Rossi Fanelli F. and Muscaritoli M., The role of docosahexaenoic acid (DHA) in the control of obesity and metabolic derangements in breast cancer, International Journal of Molecular Science. 17(4), 505 (2016).

[42] Van Dam FS. and Sternsward J., Quality of life assessment in cancer, Pharmacoeconomics. 4(4), 308-309 (1993).

[43] Ngoan LT., Mizoue T., Fujino Y., Tokui N. and Yoshimura T., Dietary factors and stomach cancer mortality, British Journal of cancer. 87(1), 37-42 (2002).

[44] Miles EA. and Calder PC., Influence of marine n-3 polyunsaturated fatty acids on immune function and a systematic review of their effects on clinical outcomes in rheumatoid arthritis. British Journal of Nutrition. 107, S171-184 (2012).

[45] Proudman SM., Cleland LG., Metcalf RG., Sullivan TR., Spargo LD. and James MJ., Plasma n-3 fatty acids and clinical outcomes in recent-onset rheumatoid arthritis. British Journal of Nutrition. 114,885-890 (2015).

[46] Calder PC., Polyunsaturated fatty acids and inflammatory processes: New twists in an old tale, Biochemistry. 91, 791-795 (2009).

[47] Freund-Levi Y., Eriksdotter-Jonhagen M., Cederholm T., Basun H., Faxen-Irving G., Garlind A., Vedin I., Vessby B., Wahlund LO. and Palmblad J., Omega-3 fatty acid treatment in 174 patients with mild to moderate Alzheimer disease, Journal Archives of Neurology. 63(10), 1402-1408 (2006).

[48] Tully AM., Roche HM., Doyle R., Fallon C., Bruce I., Lawlor B., Coakley D. and Gibney MJ., Low serum cholesterol ester docosahexaenoic acid levels in Alzheimer's disease: a case control study, British journal of nutrition. 89(4), 483-489 (2003).

[49] Gu Y., Nieves JW., Stern Y., Luchsinger JA. and Scarmeas N., Food combination and Alzheimer disease risk: a protective diet, Journal of Archives of Neurology. 67(6), 699-706 (2010). 
[50] Irving GF., Freund-Levi Y., Eriksdotter-Jonhagen M., Basun H., Brismar K., Hjorth E., Jacobs A., Botha A. and Zyl WHV., The production of eicosapentaenoic acid by representatives of the genus Mortierella grown on brewers' spent grain, Journal of Biology. 64, 871-876 (2009).

[51] Childs NM., Polyunsaturated fatty acids, Part: 1 occurrence, biological activities and applications, Chemical Technology. 24, 43-46 (1994).

[52] Velvety R. and Gill I., Polyunsaturated fatty acids, part 1: occurrence, biological activities and applications, Trends in Biotechnology. 15(10), 401-409 (1997).

[53] Kabi Pharmacia AB., World Patent No.WO9221335-A1 (1992).

[54] Asahi Denka Kogyo KK., Japanese Patent No. JP5292885-A (1993).

[55] Snow Brand Milk Products Ltd, Japanese Patent No. JP6211653-A (1994).

[56] Sandoz Nutrition Ltd, Australian Patent No. AU9513479-A (1995).

[57] Nestle SA, European Patent No. EP639333-A-1 (1995).

Citation: Naveena K C, Ramalingappa B, “Omega ( $\omega$ )-3 Polyunsaturated Fatty Acids (PUFAs) Derived from Microorganisms and Their Role in Human Health" International Journal of Research Studies In Biosciences (Ijrsb), Vol. 7, no. 7, pp. 4-12, 2019. http://Dx.Doi.org/10.20431/2349-0365.0707002

Copyright: (C) 2019 Authors. This is an open-access article distributed under the terms of the Creative Commons Attribution License, which permits unrestricted use, distribution, and reproduction in any medium, provided the original author and source are credited. 